\section{P 246 CLINICAL OUTCOME MEASURES WITHIN A SPECIALIST PALLIATIVE CARE SERVICE (SPCS): DO THEY PROVIDE ADDED VALUE?}

\author{
Catriona Mayland, ${ }^{1,2}$ Joanne Bayly ${ }^{1,2} .{ }^{1}$ Aintree University Hospital, Liverpool, UK; \\ ${ }^{2}$ Woodlands Hospice Charitable Trust
}

\subsection{6/bmjspcare-2014-000654.287}

Background Using clinical outcome measures helps support the delivery of high quality care which is amenable to critical evaluation. The project aims were to: identify potential outcome measures; pilot them within clinical settings; and provide recommendations regarding routine clinical use within a SPCS.

Methods Potential outcome measures were identified and critically evaluated. Three instruments - Palliative care Outcome Scale version 2 (POS-2); Palliative Performance Status (PPS); and Edmonton Functional Assessment Tool (EFAT) - were further assessed in a 2 month pilot within in-patient; out-patient; day therapy and outreach services. Each instrument was assessed on two consecutive occasions (unless the patient died or was discharged).

Results From 97 patients, 52 (54\%) were female and mean age was 68 years (range 27-89 years). POS-2 scores ranged from 127 (total score $40=$ very symptomatic and distressed) with highest scores present for in-patients. Seven patients had POS-2 score $>20$. All expressed high levels of psychological distress for themselves and their family.

Seventeen (73.9\%) of the 25 in-patients had a low PPS score $(<50 \%=$ considerable assistance needed for self-care). For 9 in-patients with a length of stay $>14$ days, all bar one had a low PPS score on admission.

EFAT scores showed highest levels of dysfunction were for outreach and in-patients. EFAT's ability to sufficiently discriminate clinically meaningful variations in function within each care setting limited its recommendation for regular use.

Day therapy patients tended to have the lowest POS-2 and EFAT scores.

Conclusions Following this project, POS-2 and PPS have been incorporated into routine clinical practice within the day therapy unit and more recently in-patient unit. Initial reflections suggest they aid multi-disciplinary team working and highlight particular patient concerns requiring interventions. PPS scores could potentially be linked with length of stay to help anticipate patients with particular complexity and dependency. 